\author{
Classification \\ Physics Abstracts \\ 0.660
}

\title{
ÉTUDE GÉNÉRALE DES PERFORMANCES DES DISJONCTEURS A OUVERTURE MÉCANIQUE RAPIDE POUR COURANTS INTENSES
}

\author{
C. RIOUX, F. RIOUX-DAMIDAU \\ Groupe électrotechnique et fusion contrôlée \\ Laboratoire de physique des plasmas $(*)$ \\ Université de Paris XI, 91405 Orsay, France
}

(Reçu le 25 juin 1973, révisé le 2 janvier 1974)

\begin{abstract}
Résumé. - On étudie les performances des disjoncteurs dits à rupture mécanique en vue d'effectuer un transfert rapide d'énergie d'une bobine de stockage vers une charge d'impédance variable. Les pertes Joule durant le temps de stockage et l'énergie dépensée pour ouvrir le disjoncteur sont comparées à l'énergie transférée en fonction des divers paramètres définissant l'interrupteur. On en déduit les meilleures conditions de fonctionnement de ces dispositifs. Les valeurs maximales admissibles des temps de stockage et de transfert sont données, montrant en particulier que des temps de transfert de l'ordre de la $\mu$ s peuvent être obtenus.
\end{abstract}

\begin{abstract}
The performance of circuit-breakers that open mechanically are studied for rapid energy transfer from a storage coil into a varying impedance load. The energy lost during storage and to open the switch are systematically compared with the transferred energy as a function of the various parameters defining the switch. Optimised operating conditions are derived. Extremum storage and transfer times are given. It is shown that such devices can provide transfer times of the order of $1 \mu \mathrm{s}$.
\end{abstract}

1. Introduction et exposé du problème. - Pour un certain nombre d'applications présentes ou futures, telles que l'alimentation de lasers de puissance, de souffleries à rafales, d'expériences de fusion contrôlée, il est nécessaire de mettre en œuvre des sources d'énergie électrique impulsionnelles de grande puissance instantanée. Le seul procédé couramment utilisé aujourd'hui consiste à employer des batteries de condensateurs qui sont hélas tout à la fois lourdes, coûteuses et encombrantes car elles stockent l'énergie à l'aide d'un procédé peu efficace : le champ électrostatique. Aussi, lorsque les énergies à mettre en œuvre sont importantes (par exemple quelques mégajoules), d'autres procédés de stockage plus efficients ont souvent été proposés : électrochimique, cinétique, magnétique, etc. Grosso modo, tous ces dispositifs accumulent par unité de masse des énergies de 100 à 1000 fois celles des condensateurs mais difficiles à libérer rapidement.

Les stockages électrochimique ou cinétique sont déjà employés avec succès lorsque la durée de l'impulsion est supérieure à $10^{-1} \mathrm{~s}$, voire $10^{-2} \mathrm{~s}$. Mais certaines applications (expériences de fusion contrôlée de type $\theta$-pinch, focus, etc.) exigent des impulsions plus courtes proches des possibilités limites des grandes

(*) Laboratoire associé au C. N. R. S. batteries de condensateurs $\left(10^{-6} \mathrm{~s}\right)$. Alors, deux procédés restent raisonnablement envisageables: les générateurs à explosif et le stockage magnétique. En ce qui concerne les générateurs à explosif, le coût d'exploitation est élevé car chaque impulsion s'accompagne de la destruction d'une partie de l'appareillage ; leur utilisation intensive présenterait par suite des problèmes considérables. Le stockage magnétique paraît alors le seul procédé pratique permettant à priori de délivrer des impulsions courtes de grande énergie avec des coûts d'investissement et d'exploitation nettement plus faibles que les condensateurs.

Aussi, nous nous sommes intéressés aux conditions de faisabilité de tels générateurs, lesquels sont constitués par l'association de deux éléments principaux : une bobine magnétique et un disjoncteur. Si la réalisation des bobines (classiques ou supraconductrices) ne présente plus aujourd'hui de difficultés majeures, la construction du disjoncteur à la fois rapide et puissant indispensable se heurte toujours à des problèmes fort difficiles. Les caractéristiques des sources à stockage magnétique sont finalement limitées par notre savoir-faire en matière de disjoncteur, lequel savoir-faire n'est toujours pas suffisant pour rendre ces sources concurrentielles des meilleurs condensateurs. 
Dans l'état actuel de la technique, il existe de nombreux types de disjoncteurs mais aucun n'est réellement adapté au transfert d'une énergie importante en un temps nettement inférieur à la ms. Par exemple, les disjoncteurs de haute puissance placés sur les réseaux de distribution électrique [1] coupent des courants alternatifs élevés lors de leur passage à zéro en seulement quelques centaines de $\mu$ s. Les interrupteurs à supra conducteurs [2-3] peuvent commuter des courants en quelques $\mu$ s mais transfèrent effectivement l'énergie en des temps de l'ordre de la ms. Pour des transferts plus rapides, on s'adresse à des interrupteurs spéciaux qui consistent tous à rompre une chaîne conductrice, puis à placer un isolant entre les lèvres de la coupure. Un cas particulier de cette catégorie est fourni par les disjoncteurs à vide [4-5] ; ils sont très fréquemment utilisés pour des applications de ce genre mais ne permettent pas de couper des courants dépassant nettement $10 \mathrm{kA}$. Les performances souhaitées sont seulement atteintes lorsqu'on utilise des jets d'isolant produits par charge creuse [6]. Malheureusement, l'énergie dépensée pour créer ce jet est très importante. Remarquons d'autre part que pour tous ces disjoncteurs, les caractéristiques de fonctionnement dépendent très peu du temps de stockage de l'énergie, c'est-à-dire du temps de passage du courant lorsqu'ils sont fermés. Si cette dernière contrainte n'existe pas, on connaît un procédé de coupure très efficace valable tout à la fois pour les courants très forts et les temps très courts : les fils explosants. Dans ce dernier cas, hélas, le temps de passage initial du courant ne peut excéder 10 fois le temps de transfert d'énergie [7-8].

Finalement, il n'existe actuellement aucun disjoncteur qui puisse être associé à une bobine de stockage d'ênergie magnétique pour former une source d'énergie susceptible de concurrencer ou dépasser les meilleurs bancs de condensateurs.

Aussi, nous avons repris le problème de la faisabilité des disjoncteurs rapides : est-elle possible et par quels moyens?

Pour préciser ce problème, rappelons qu'un disjoncteur est un dispositif à impédance variable qui fonctionne grosso-modo en trois étapes. Tout d'abord, son impédance est faible; il supporte un courant important durant un temps long en ne présentant à ses bornes qu'une faible différence de potentiel. Ensuite, son impédance croît rapidement, le courant étant transféré vers le circuit d'utilisation. Par ailleurs, durant le transfert du courant ou juste après, il apparaît à ses bornes une tension élevée qui engendre le transfert de l'énergie en un temps court. Si les deux dernières phases se recoupent, c'est-à-dire si la surtension apparaît pendant le transfert du courant, le disjoncteur doit absorber une énergie importante: il en est ainsi chaque fois que la charge d'utilisation est fixe. Nous montrerons qu'au contraire, si la charge est elle-même constituée par une impédance variable, petite pendant le transfert du courant et croissant ultérieurement, les deux dernières étapes peuvent être disjointes. Le disjoncteur n'absorbe plus que de faibles énergies, ce qui facilite beaucoup son fonctionnement. Etant donné que l'on peut toujours rendre variable une impédance fixe quelconque en lui associant un fil explosant en parallèle, le problème peut donc être ramené à la réalisation de disjoncteurs absorbant peu d'énergie.

Nous avons alors examiné systématiquement tous les types d'interrupteurs répondant à cette dernière condition. De nombreuses similitudes de fonctionnement étant apparues, nous avons pensé qu'il. était bon d'étudier au préalable le type qui paraissait le plus prometteur : le disjoncteur à déplacement mécanique de conducteur (la comparaison avec les autres procédés sera publiée ultérieurement). Bien que la technologie à mettre en œuvre soit souvent compliquée, le schéma de principe est très simple : on enlève un morceau de conducteur que l'on remplace par de l'isolant. Ce schéma nous a permis de chiffrer les temps de stockage et de transfert qu'il est possible d'obtenir lorsqu'on impose que l'énergie dépensée pour faire fonctionner l'interrupteur ne soit qu'une faible fraction de l'énergie transférée. Cette étude met en évidence l'influence des divers paramètres du système (natures du conducteur et de l'isolant, dimensions, vitesse de remplacement du métal par l'isolant, caractéristique de variation de l'impédance de la charge). Nous conclurons en donnant les valeurs optimales de ces paramètres et les possibilités limites de ces disjoncteurs.

2. Description formelle du fonctionnement des disjoncteurs. - De façon générale, les disjoncteurs sont essentiellement utilisés pour transférer vers une charge $C$ tout ou partie de l'énergie magnétique $W_{\mathbf{B}}$ stockée dans une bobine magnétique B. Le montage du circuit est alors celui de la figure 1 ; les tensions et

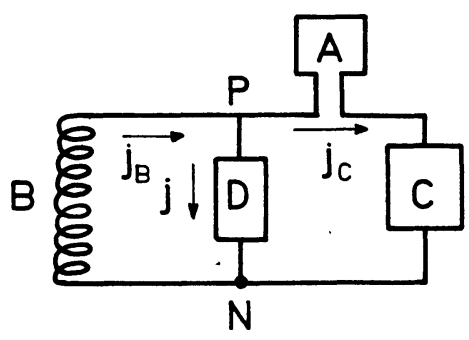

FIG. 1. - Circuit de transfert d'énergie. Le disjoncteur D étant fermé, l'énergie est stockée initialement dans la bobine $B$. Le courant dans $\mathrm{D}$ est ensuite annulé à l'aide du dispositif $\mathrm{A}$. Enfin l'ouverture de $\mathrm{D}$ transfère l'énergie dans la charge $C$.

courants sont représentés en fonction du temps sur la figure 2.

Tout d'abord, durant le long temps de stockage $T_{0}$, la bobine B est parcourue par la totalité du courant, le disjoncteur D étant fermé. Puis, on ouvre ce dernier. Si cette ouverture s'effectuait sans précautions, il se formerait entre les bornes du circuit un arc d'extra- 


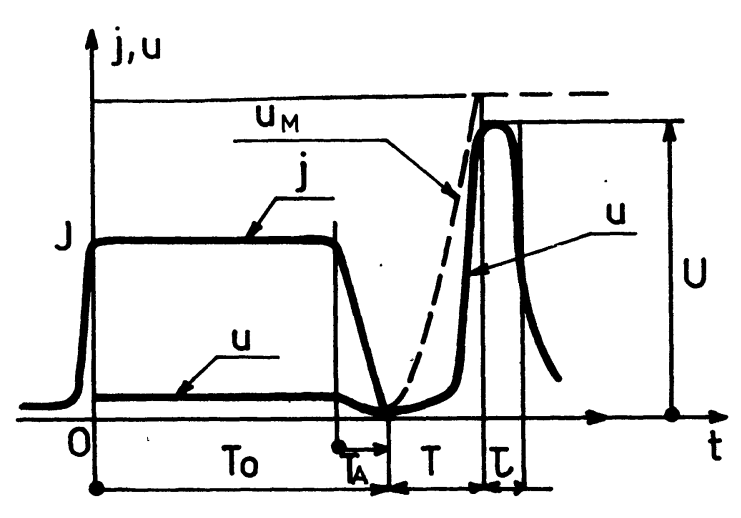

Fig. 2. - Variation en fonction du temps du courant $j$ dans le disjoncteur et de la tension $u$ à ses bornes. En pointillé, nous avons tracé la tension limite $U_{M}$ d'isolement du disjoncteur.

rupture qui dissiperait de l'énergie et polluerait l'isolant. Pour éviter cela, on annule le courant dans D juste avant son ouverture par un dispositif extérieur $\mathrm{A}$ placé en série avec la charge. Appelons $T_{\mathrm{A}}$ la durée de cette opération (même lorsqu'elle est effectuée par simple augmentation de l'impédance de D), $L_{\mathrm{co}}$ et $R_{\text {Co }}$ l'inductance et la résistance de la charge pendant le temps $T_{0}, L_{\mathrm{B}}$ l'inductance de la bobine $\mathrm{B}$ (nous supposons la résistance de cette dernière négligeable).

$\mathrm{Si}$

$$
T_{\mathrm{A}} \ll \frac{L_{\mathrm{CO}}}{R_{\mathrm{CO}}} \quad \text { et } \quad L_{\mathrm{CO}} \ll L_{\mathrm{B}},
$$

l'énergie dépensée pour effectuer l'annulation du courant est en pratique purement inductive et négligeable comparée à l'énergie stockée $W_{\mathbf{B}}$. Nous supposerons qu'il en est ainsi.

Après avoir effectué ce transfert du courant, on court-circuite A et l'on ouvre le disjoncteur en un temps $T$. La tension $u_{\mathrm{M}}(t)$ que $\mathrm{D}$ est susceptible de supporter sans claquage croît avec le temps. La bobine $\mathbf{B}$ déverse son énergie dans $\mathrm{C}$ en un temps $\tau$, durant lequel le disjoncteur est soumis alors à une pointe de tension d'amplitude $U$. En ce qui concerne ce transfert de l'énergie, les caractéristiques en sont différentes suivant que l'impédance de $\mathrm{C}$ reste fixe (donc égale à $L_{\mathrm{Co}}$ et $R_{\mathrm{CO}}$ ) ou est variable avec le temps (en prenant les valeurs $L_{\mathrm{C}}$ et $R_{\mathrm{C}}$ ).

Dans le cas où l'impédance est fixe, elle est essentiellement résistive puisque $L_{\mathrm{CO}}$ est négligeable (cf. (2)). L'énergie $W_{\mathrm{B}}$ est dissipée par effet Joule dans $R_{\mathrm{CO}}$ pendant le temps $\tau \simeq L_{\mathrm{B}} / R_{\mathrm{CO}}$. Compte tenu des hypothèses faites ci-dessus, nous avons :

$$
\tau \gg \frac{L_{\mathrm{CO}}}{R_{\mathrm{CO}}} \gg T_{\mathrm{A}} .
$$

Le temps de dissipation de l'énergie dans C, c'est-àdire le temps de récupération de l'énergie de $\mathrm{B}$ par $\mathrm{C}$, est beaucoup plus long que le temps $T_{\mathrm{A}}$ de transfert du courant.

Si, au contraire, l'impédance de la charge est croissante, le transfert de l'énergie sera plus rapide que précédemment. En particulier, si on désire que le temps de transfert $\tau$ soit très court, nous devrons provoquer pendant ce temps $\tau$ une très rapide croissance soit de $R_{\mathrm{C}}$, soit de $L_{\mathrm{C}}$, telle que :

$$
\Delta R_{\mathrm{C}} \simeq L_{\mathrm{B}} / \tau
$$

ou

$$
\Delta L_{\mathrm{C}} \sim L_{\mathrm{C}} \gtrsim L_{\mathrm{B}}
$$

En ce qui concerne $R_{\mathrm{C}}$, sa variation rapide peut être obtenue en employant un fil explosant, dont la résistance est faible à froid et devient très grande lorsqu'il explose. Pour $L_{\mathrm{C}}$, il existe également de nombreux cas où sa valeur varie rapidement (expériences de physique des plasmas de type focus ou $\theta$-pinch, formage magnétique, etc...).

Finalement, la récupération rapide de l'énergie n'est possible que si l'impédance de la charge est faible lors du transfert du courant et à croissance très rapide lors de la phase suivante du transfert d'énergie. C'est pourquoi, si l'on désire effectuer un transfert rapide d'énergie dans le cas très courant où la charge est une inductance fixe quelconque $L_{C}$, il faut réaliser une charge artificiellement variable en associant par exemple à $L$ un fil explosant placé en parallèle. L'annulation du courant se fait avec une faible dépense d'énergie puisque l'impédance mise en jeu dans cette étape est la résistance du fil froid et que les conditions (1) et (2) sont réalisées. Lorsque le fil explose, l'impédance vue par le disjoncteur croît ensuite brutalement jusqu'à la valeur $L_{\mathrm{C}}$.

Le passage d'une impédance de charge faible à une impédance élevée sera donc la plupart du temps déclenché par le transfert du courant. Si on regarde alors l'évolution en fonction du temps de l'impédance vue par le disjoncteur, on peut grosso-modo définir un temps $T_{l}$ pendant lequel sa valeur reste petite puis un temps $\tau$ pendant lequel elle est grande. Pendant le temps $T_{l}$, que nous appellerons " temps de latence ", la tension aux bornes du disjoncteur est faible; elle ne devient élevée que pendant le temps $\tau$ au cours duquel s'effectue l'absorption d'énergie par la charge. Le disjoncteur n'a donc besoin d'être bien isolé qu'au bout du temps $T_{l}$. Etant donné que l'isolement maximum du disjoncteur a lieu au bout du temps $T$ (cf. Fig. 2), il sera donc bon de choisir $T_{l} \gtrsim T$.

Finalement, les temps caractéristiques sont :

- $T_{l}$ et $\tau$ pour la charge,

- $T$ et $\tau$ pour le disjoncteur.

Dans la suite de notre étude, nous utiliserons plutôt les trois paramètres $\tau, \beta_{0}$ et $\beta$ avec :

$$
\begin{aligned}
\beta_{0} & =\frac{T_{l}}{\tau} \\
\beta & =\frac{T}{\tau} .
\end{aligned}
$$

Si l'on réalise $T_{l} \gtrsim T$, nous aurons donc $\beta_{0} \gtrsim \beta$. 
Dans le cas classique où la charge est un fil explosant, le temps $T_{l}$, qui dépend essentiellement de la densité de courant, peut varier dans de très larges limites tandis que le rapport $\beta_{0}$ reste en général petit. Si l'on admet l'hypothèse simplificatrice $T_{\mathrm{A}}=0, \beta_{0}$ est de l'ordre de quelques unités [7-8]. Dans nos exemples numériques, nous prendrons comme valeurs typiques $\beta=\beta_{0}=1$ et 5 .

Nous venons de préciser les conditions physiques formelles du fonctionnement des interrupteurs. Pour poursuivre notre étude, nous allons maintenant examiner les conditions énergétiques (rendement, énergie transférée) sur l'un des types qui nous paraît le plus prometteur : le disjoncteur à rupture mécanique.

3. Le disjoncteur à rupture mécanique : structure et conditions de fonctionnement. -3.1 DESCRIPTION GÉNÉRALE. - De manière encore générale, le fonctionnement d'un disjoncteur ne sera acceptable pour un utilisateur que si les pertes provoquées à la fois par la commande de son ouverture et par l'effet joule pendant le passage initial du courant sont notablement inférieures à l'énergie transférée sur la charge. Considérons ce problème pour les disjoncteurs à rupture mécanique [9-10] dont nous donnons le schéma de principe figure 3. Remarquons bien que ce dessin très simple, destiné à appuyer notre étude théorique, recouvre en fait de nombreuses structures technologiques très différentes et malheureusement plus complexes que ce schéma.
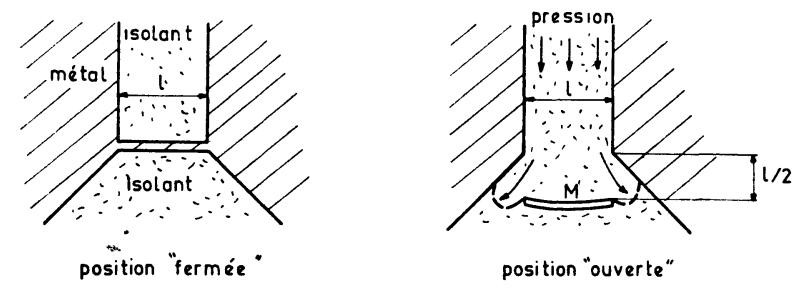

position "ouverte"

Fig. 3. - Schéma de principe du disjoncteur mécanique.

Lorsqu'un tel disjoncteur est fermé, il est constitué d'un simple conducteur parcouru par un courant $J$ durant le temps $T_{0}$; il présente une partie rétrécie $M$ de section régulière $\mathrm{s}$ de longueur $l$. Après annulation du courant, on applique sur la partie supérieure de l'isolant une surpression convenable qui entraîne la partie $M$ du conducteur. L'intervalle ainsi libéré est rempli d'un isolant supportant alors un champ électrique moyen $E$; nous admettons que l'isolement est assuré au bout du temps $T$ lorsque le morceau $M$ s'est écarté du conducteur d'une distance égale à $l / 2$. Ce morceau de masse spécifique $\bar{\omega}$ a alors une vitesse $v$ et une énergie cinétique $\frac{1}{2} \bar{\omega} v^{2} l s$ et l'on peut écrire avec (7) :

$$
\frac{l}{2} \simeq v T=\beta v \tau .
$$

Lors de l'ouverture du circuit, une tension $U \doteq E l$ apparaît aux bornes du disjoncteur (c'est-à-dire aux bornes de la coupure) tandis que l'énergie absorbée par la charge vaut $W \simeq U J_{\mathrm{c}} \tau$. Si $i$ est la densité de courant dans $\mathrm{M}$ avant coupure, on a $J_{\mathrm{c}}=i$ is et par suite $W=$ Eirls.

Pour assurer le transfert de l'énergie $W$, deux types de pertes apparaissent : l'énergie Joule $W_{\mathrm{J}}$ dissipée et l'énergie $W_{\mathrm{R}}$ destinée à provoquer la cassure.

L'énergie Joule se dissipe presque uniquement dans $\mathrm{M}$ durant le temps $T_{0}$. Si $\rho$ est la résistivité de $\mathrm{M}$ à chaque instant, on a :

$$
W_{\mathrm{J}}=\int_{0}^{T_{0}} \rho i^{2} l \mathrm{~s} \mathrm{~d} t
$$

Pour remplacer le morceau M par de l'isolant, on doit rompre le conducteur et mettre en vitesse $M$ et l'isolant. L'énergie nécessaire pour provoquer la cassure est en général négligeable comparée à l'énergie cinétique $W_{\mathrm{c}}=\frac{1}{2} \bar{\omega} v^{2} l \mathrm{~s}$ de $\mathrm{M}$. Par ailleurs, la quantité d'isolant qui se déplace en même temps que $M$ est à peu près proportionnelle au volume de $\mathbf{M}$. Aussi, nous pourrons écrire que, pour assurer l'ouverture de $\mathbf{M}$, il faudra fournir une énergie :

$$
W_{\mathbf{R}}=\gamma W_{\mathrm{c}}
$$

où $\gamma$ est de l'ordre de quelques unités.

Notons que toutes les énergies qui interviennent dans le problème considéré sont proportionnelles au volume $l \mathrm{~s}$ de $\mathrm{M}$; aussi, nous ne nous intéresserons désormais qu'aux énergies correspondant au volume unité :

$$
\begin{aligned}
& w=E i \tau \\
& w_{\mathrm{J}}=\int_{0}^{T_{\mathrm{o}}} \rho i^{2} \mathrm{~d} t \\
& w_{\mathrm{R}}=\gamma w_{\mathrm{c}}=\gamma \cdot \frac{1}{2} \bar{\omega} v^{2}=\frac{\gamma \bar{\omega}}{8 \beta^{2}} \cdot \frac{l^{2}}{\tau^{2}} .
\end{aligned}
$$

Les pertes volumiques totales valent alors :

$$
w_{\mathbf{P}}=w_{\mathbf{R}}+w_{\mathbf{J}} .
$$

Si nous appelons :

$$
\eta_{\mathrm{J}}=\frac{w_{\mathrm{J}}}{w} \quad \text { et } \quad \eta_{\mathrm{R}}=\frac{w_{\mathrm{R}}}{w}
$$

les pertes relatives dues à l'effet Joule et à la rupture, les pertes relatives totales valent alors :

$$
\eta=\frac{w_{\mathrm{P}}}{w}=\eta_{\mathrm{J}}+\eta_{\mathrm{R}} .
$$

Ces pertes dépendent de $w_{\mathrm{R}}$ qui est fixé en partie par des conditions extérieures, de $w$ qui est une caractéristique fondamentale des possibilités du disjoncteur et de $w_{\mathrm{J}}$ que nous allons évaluer maintenant. Le problème consistera ensuite à chercher les conditions de fonctionnement pour lesquelles $\eta$ reste faible. 
3.2 Evaluation De $w_{\mathrm{J}}$ - - Par suite du passage du courant dans le disjoncteur fermé, la température $\theta$ de la partie rétrécie $M$ varie. Elle est donnée par l'équation classique :

$$
\rho i^{2}+k \Delta \theta=\bar{\omega} c \frac{\mathrm{d} \theta}{\mathrm{d} t} .
$$

Le coefficient de conductibilité thermique $k$ et la chaleur spécifique $c$ sont pratiquement indépendants de la température. $\mathrm{La}$ résistivité $\rho$ varie suivant la loi approximative [13]:

$$
\rho=\rho_{0} \theta / \theta_{0} .
$$

Nous pouvons donc calculer $w_{\mathrm{J}}$ à partir de (10), ce que nous avons effectué dans les deux cas extrêmes où la diffusion de la chaleur est négligeable $(k \Delta \theta \simeq 0)$ et où un état stationnaire est atteint $(\bar{\omega} c \mathrm{~d} \theta / \mathrm{d} t \simeq 0)$ [11]. Nous avons alors montré que $w_{\mathbf{J}}$ pouvait toujours s'écrire sous la forme :

$$
w_{\mathrm{J}}=\alpha \rho_{0} i^{2} T_{0} .
$$

Le paramètre $\alpha$ ainsi défini représente le rapport entre la valeur moyenne de la résistivité au cours du temps $T_{0}$ et la valeur $\rho_{0}$ de la résistivité à l'instant initial $(\alpha \geqslant 1)$.

En admettant que la température maximum admissible est $\theta_{\mathrm{M}}=e \cdot \theta_{0}(e=2,72)$, on montre que $\alpha$ est grosso-modo au plus égal à 2 et on peut calculer les valeurs maximales $i_{\mathrm{M}}$ ou $i_{\mathrm{M}}^{d}$ de la densité de courant, $w_{\mathrm{JM}}$ et $\left(w_{\mathrm{J}}^{d}\right)_{\mathrm{M}}$ de la densité d'énergie Joule.

Pratiquement, on distingue les deux cas en comparant la demi-longueur $l / 2$ du conducteur à la longueur de diffusion de la chaleur durant le temps $T_{0}$ [12] :

$$
L=\left(\frac{k T_{0}}{\bar{\omega} c}\right)^{1 / 2}
$$

(on suppose l'épaisseur de $\mathrm{M}$ très petite comparée à $l$ ).

Si $l / 2 L \gg 1$, la chaleur dégagée ne diffuse pas. Elle sert presque uniquement à élever la température locale du conducteur. On montre que

$$
1 \leqslant \alpha=\frac{w_{0}}{\rho_{0} i^{2} T_{0}}\left(\exp \frac{\rho_{0} i^{2} T_{0}}{w_{0}}-1\right) \leqslant(e-1)
$$

$\left(\right.$ avec $\left.w_{0}=\bar{\omega} c \theta_{0}\right)$ et que :

$$
w_{\mathrm{JM}}=(e-1) w_{0} .
$$

Si $l / 2 L \ll 1$, la plus grande partie de la chaleur dégagée est évacuée hors la région $M$ vers les parties massives. Alors :

$$
\begin{gathered}
1 \leqslant \alpha=\lambda \operatorname{tg} \lambda \leqslant 2,1\left(\operatorname{avec} \lambda=\frac{2}{l}\left(\frac{k \theta_{0}}{\rho_{0} i^{2}}\right)^{1 / 2}\right) \\
\left(w_{\mathrm{J}}^{d}\right)_{\mathrm{M}}=12,6 \frac{L^{2}}{l^{2}} w_{0} .
\end{gathered}
$$

\subsection{EXPRESSION DE $\eta$. - En remplaçant $i$ par $w / E \tau$}

(cf. (9)) dans l'expression générale (17), nous pouvons aussi écrire :

$$
w_{\mathrm{J}}=\alpha\left(\frac{\rho_{0}}{E^{2}} \cdot \frac{T_{0}}{\tau^{2}}\right) w^{2} .
$$

Reportant (23) dans la définition (14) de $\eta$, nous obtenons :

$$
\eta=\alpha\left(\frac{\rho_{0}}{E^{2}} \cdot \frac{T_{0}}{\tau^{2}}\right) w+\frac{w_{\mathrm{R}}}{w} .
$$

Nous venons ainsi d'exprimer les pertes relatives $\eta$ en fonction des conditions d'utilisation. Nous allons maintenant étudier en détail cette expression.

4. Diagramme $\eta(w)$. - 4.1 Tracé. - Les pertes relatives $\eta$ sont fonction de nombreuses variables :

$$
\eta=\eta\left(w, \frac{\rho_{0}}{E^{2}} \cdot \frac{T_{0}}{\tau^{2}}, w_{\mathrm{R}}, \alpha\right) .
$$

Pour une utilisation donnée, $\frac{\rho_{0}}{E^{2}} \cdot \frac{T_{0}}{\tau^{2}}$ et $w_{\mathrm{R}}$ sont généralement fixés et $\alpha$ est un paramètre correctif variant peu.

Aussi, sur la figure 4, nous avons porté $\eta$ en fonction de $w$ en coordonnées logarithmiques pour $\frac{\rho_{0}}{E^{2}} \frac{T_{0}}{\tau^{2}}$ et $w_{\mathrm{R}}$ donnés. La courbe en traits pleins correspond au cas où $\alpha$ reste constamment égal à 1 ; elle possède deux asymptotes qui se coupent en un point $G$ et qui sont parallèles aux bissectrices $O X$ et $O Y$ de $(O w \eta)$. Lorsque $\alpha$ devient différent de 1 , c'est-à-dire lorsque le morceau $\mathbf{M}$ du conducteur ne garde pas une température constante durant le temps $T_{0}$, cette courbe est légèrement modifiée ; nous avons indiqué en traits pointillés cette modification dans le cas particulier où $w_{0} \gg w_{\mathrm{R}}$.

Pour faire apparaitre simplement certains paramètres de base, plaçons-nous dans le référentiel $(O X Y)$. Si $K$ est un point quelconque de coordonnées logarith-

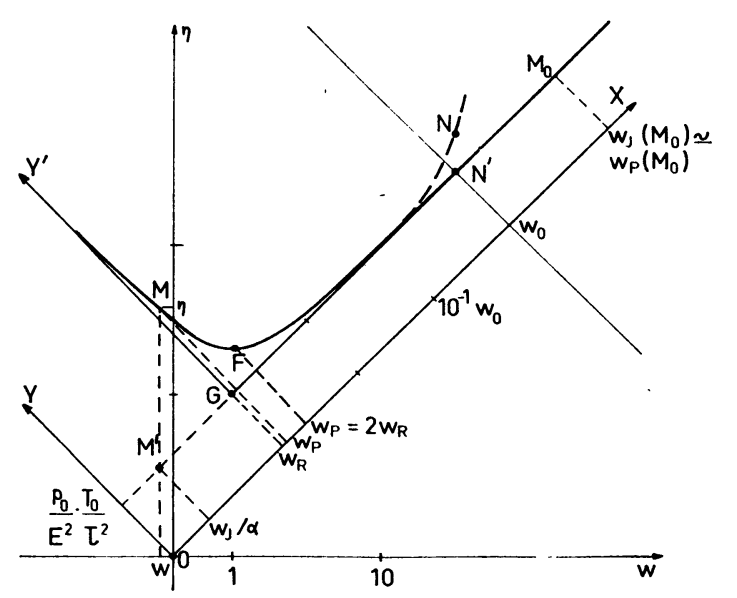

Fig. 4. - Diagramme général des pertes relatives $\eta$ en fonction de la densité d'énergie transférable $w$ lorsque la densité d'énergie de rupture $w_{\mathrm{R}}$ et le paramètre $\frac{\rho_{0}}{E^{2}} \cdot \frac{T_{0}}{\tau^{2}}$ sont donnés. 
miques $w_{K}$ et $\eta_{K}$ dans le référentiel $(O w \eta)$, ses coordonnées logarithmiques dans le nouveau référentiel sont :

$$
\begin{aligned}
X_{K} & =\eta_{K} w_{K} \\
Y_{K} & =\frac{\eta_{K}}{w_{K}} .
\end{aligned}
$$

En particulier, les coordonnées de $G$ valent, compte tenu de (24) et (25) :

$$
\begin{aligned}
& X_{G}=\eta_{G} w_{G}=w_{R} \\
& Y_{G}=\frac{\eta_{G}}{w_{G}}=\frac{\rho_{0}}{E^{2}} \cdot \frac{T_{0}}{\tau^{2}} .
\end{aligned}
$$

Remarquons que pour $\alpha=1$, la connaissance de $G$ détermine entièrement la courbe $\eta(w)$. Pour $\alpha \neq 1$, le point $G$ dont les coordonnées dans $(O X Y)$ correspondent aux deux paramètres fondamentaux $w_{R}$ et $\frac{\rho_{0}}{E^{2}} \cdot \frac{T_{0}}{\tau^{2}}$ n'est plus un point de concours d'asymptotes mais il conserve l'intérêt de représenter ces paramètres.

Considérons maintenant un point de fonctionnement $M$ situé sur la courbe $\eta=\eta(w, \alpha)$ pour laquelle $G$ est connu et montrons que la plupart des grandeurs physiques correspondant à ce fonctionnement sont définies graphiquement de façon simple. En effet :

- Les paramètres $w_{\mathrm{R}}$ et $\frac{\rho_{0}}{E^{2}} \cdot \frac{T_{0}}{\tau^{2}}$ sont les coordonnées de $G$ dans $(O X Y)$.

- $\eta$ et $w$ sont les coordonnées de $M$ dans $(O w \eta)$.

- L'abscisse de $M$ dans (OXY) est, d'après (25) et (14) :

$$
(X)_{M}=\eta w=w_{P} .
$$

- Si $M^{\prime}$ est l'intersection de la droite $G X^{\prime}$ parallèle à $O X$ et de la verticale issue de $M$, un calcul simple montre que :

$$
X_{M^{\prime}}=\frac{w_{\mathrm{J}}}{\alpha}
$$

- Enfin, si nous posons :

$$
w_{t}^{2}=\frac{E^{2}}{\rho_{0}} \cdot \frac{k}{c} \frac{\gamma}{2 \beta^{2}}
$$

il vient

$$
\frac{l}{2 L}=\frac{w_{G}}{w_{t}}
$$

Il suffit donc de comparer la coordonnée $w_{G}$ de $G$ à la valeur $w_{\mathrm{t}}$ pour connaître les conditions d'échauffement :

$$
\text { si } \frac{l}{2 L}>1 \text { ou } w_{G}>w_{\mathrm{t}} \text {, la chaleur ne peut s'évacuer }
$$
du conducteur ;

$$
\text { si } \frac{l}{2 L}<1 \text { ou } w_{G}<w_{\mathrm{t}} \text {, la chaleur diffuse. }
$$

4.2 Limitations par Échauffement. Diagramme GÉNÉRAL. - Lórsqu'on décrit une courbe $\eta(w, \alpha)$ de gauche à droite, $w_{\mathrm{J}}$ (de même que $w$ et $i$ ) augmente. Or nous avons vu que les densités d'énergie Joule acceptables étaient limitées par les valeurs

$$
w_{\mathrm{J} M}=(e-1) w_{0}
$$

lorsque la chaleur ne pouvait pas diffuser et

$$
\left(w_{\mathrm{J}}^{d}\right)_{M}=12,6 \frac{L^{2}}{l^{2}} w_{0}
$$

lorsqu'elle diffusait. Par suite les courbes s'arrêtent en un point situé à gauche ou à droite du minimum $F$ suivant que la valeur maximale de $w_{\mathrm{J}}$ est inférieure ou supérieure à $2 w_{\mathrm{R}}$.

Nous avons alors recherché de façon précise le lieu des points limites et nous en avons déduit le diagramme de la figure 5. Sur celui-ci, nous avons porté les diverses courbes $\eta(w)$ correspondant à un interrupteur et une charge donnés (isolant, conducteur, $l, \gamma$ et $\beta$ fixés) pour diverses valeurs de $T_{0} / \tau^{2}$ et de $w_{\mathrm{R}}$, c'est-à-dire pour divers points $G$.

Ce diagramme fait intervenir les deux droites $\mathcal{C}$ et $\mathfrak{D}$ d'équations :

$$
\begin{aligned}
X & =w_{0} \\
\eta w^{3} & =1,45 w_{0} w_{\mathrm{t}}^{2}
\end{aligned}
$$

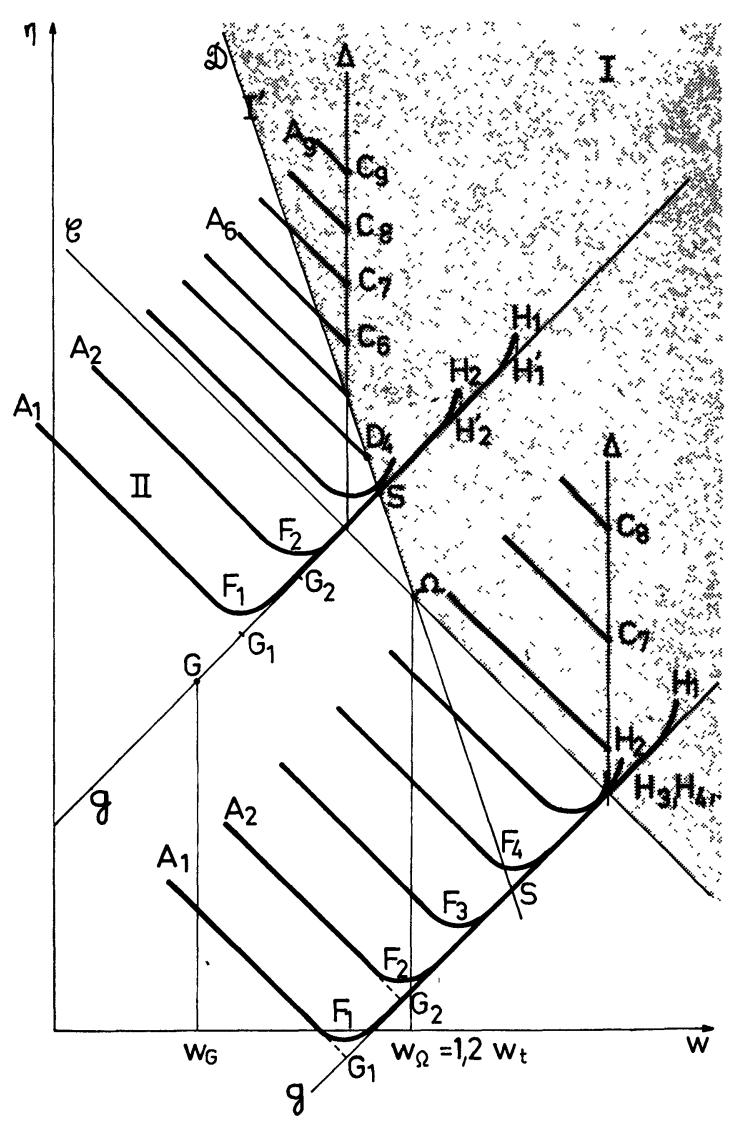

Fig. 5. - Diagramme montrant les différentes formes de la courbe $\eta(w)$ pour les diverses valeurs possibles des paramètres

$$
\frac{\rho_{0}}{E^{2}} \cdot \frac{T_{0}}{\tau^{2}} \text { et } w_{\mathrm{R}} \text {. }
$$


qui se coupent en un point $\boldsymbol{\Omega}$ dont les coordonnées sur les différents axes valent :

$$
\begin{gathered}
X_{\Omega}=w_{0}, \quad w_{\Omega}=1,2 w_{\mathrm{t}}, \\
\eta_{\Omega}=\frac{w_{0}}{1,2 w_{\mathrm{t}}}, \quad Y_{\Omega}=\frac{w_{0}}{1,45 w_{\mathrm{t}}^{2}} .
\end{gathered}
$$

Les deux droites $\mathcal{C}$ et $\mathfrak{D}$ délimitent alors deux zones, la zone I située à droite de $\mathfrak{D}$ et $\mathcal{C}$ et la zone II complémentaire de I.

Remarquons que la position de $\boldsymbol{\Omega}$ est déterminée par les matériaux constituant l'interrupteur et le paramètre d'utilisation $\beta=T / \tau$. Elle permet de repérer en particulier les conditions de transfert thermique. En effet, comme $w_{\Omega} \simeq w_{t}$, on a : $l / 2 L \simeq w_{G} / w_{\Omega}$. Si $w_{G}<w_{\Omega}$, la chaleur dégagée diffuse hors du conducteur. Au contraire, si $w_{G}>w_{\Omega}$, cette diffusion est négligeable.

Le diagramme montre clairement les deux formes typiques des courbes $\eta(w)$ :

- les quasi-droites $A C$ (ou $A D$ ) lorsque le point limite $C$ (ou $D$ ) est à gauche de $F$. Alors, $w_{\mathbf{J} M}$ ou $\left(w_{\mathrm{J}}^{d}\right)_{M}$ est très inférieur à $w_{\mathrm{R}}$,

- les courbes avec minimum $A F H$ lorsque le point limite $M$ est à droite de $F$ et que $w_{\mathrm{J} M}$ ou $\left(w_{\mathrm{J}}^{d}\right)_{M}$ est très supérieur à $w_{\mathrm{R}}$.

Il montre aussi comment la courbe $\eta(w)$ se déforme lorsque $w_{\mathrm{R}}$ varie tandis que $T_{0} / \tau^{2}$ reste constant (c'est-à-dire lorsque le point $\mathrm{G}$ se déplace sur une droite $\mathcal{G}$, parallèle à $O X$ ).

La construction des points limites des courbes $\eta(w)$ est assez complexe [11] :

Tant que $G$ est dans la zone I, le point limite $C$.est en général situé sur une droite $\Delta$ qui est la verticale passant par le point de concours des droites $\mathcal{C}$ et $\mathbf{G}$. Il est situé sur la droite $\mathfrak{D}$ dans le cas particulier où le point $D$ d'intersection de $\mathfrak{D}$ avec $\eta(w)$ est à droite du point d'intersection de $\Delta$ avec $\eta(w)$; il ne peut en être ainsi que si $Y_{G}>Y_{\Omega}$. Dans ce dernier cas, nous dirons que $D$ est dans la zone $I^{\prime}$, qui sera donc la demi-droite supérieure $\Omega \mathcal{D}$, alors que le point $C$ est dans la zone I. Si $G$ est dans la zone II, les courbes sont du type $A F H$. Si $S$ est l'intersection de $\mathcal{G}$ et $\boldsymbol{D}$ on a :

$$
\overline{\mathbf{S}} \mathbf{G}=-\overline{\mathbf{S}} \mathbf{H}^{\prime}
$$

où $H^{\prime}$ est la projection de $H$ sur $\mathcal{G}$, à moins que le point $H^{\prime}$ ainsi construit ne soit dans la zone I, auquel cas $H$ est sur $\Delta$.

Remarquons que si un point de fonctionnement $M$ est situé sur une des courbes $\eta(w)$ assimilables à une quasi-droite, le point limite $C$ (ou $D$ ) situé sur $\Delta$ (ou $D)$ représente la meilleure utilisation des possibilités de l'interrupteur puisqu'en augmentant la densité de courant jusqu'à atteindre $C$ (ou $D)$ on augmente à la fois le rendement et la densité d'énergie transférée. Le point $F$ des courbes de type $A F H$ possède les mêmes propriétés vis-à-vis des points de fonctionnement situés sur les portions de courbes $A F$. Inversement, lorsque le point de fonctionnement est sur $F C$ ou $F H$, accroître la densité de courant est bénéfique en ce qui concerne l'énergie transférée mais défavorable en ce qui concerne le rendement ; le choix précis du point de fonctionnement dépend alors de la valeur de $\eta$ tolérable. Ainsi, les seules parties intéressantes des courbes $\eta(w)$ en ce qui concerne l'utilisation des interrupteurs sont les points situés sur les droites $\Delta$ et $D$ et les parties $F C$ ou $F H$.

4.3 Diagramme en COORdonnées $T_{0}$ ET $\tau$. - Pour le moment, considérons seulement les points de fonctionnement du type $C$ ou $F$. A chacun de ces points, on peut associer un seul point $G$ définissant la courbe $\eta(w)$ dont ils sont issus. La donnée de $C$ ou $F$ définit donc complètement le fonctionnement de l'interrupteur. Or, on peut montrer que les coordonnées de ces points vérifient :

- en ce qui concerne $C$ :

$$
\begin{aligned}
\eta_{C} w_{C}^{3} & =w_{0} w_{\mathrm{t}}^{2}\left(\frac{l}{2 L}\right)^{2}, \\
X_{C} & =w_{\mathrm{R}}
\end{aligned}
$$

- en ce qui concerne $F$ :

$$
\begin{aligned}
& w_{F}=w_{\mathrm{t}} \cdot \frac{l}{2 L}, \\
& X_{F}=2 w_{\mathrm{R}} .
\end{aligned}
$$

Or, lorsque la nature du conducteur et celle de l'isolant sont connues et lorsque $\gamma / \beta^{2}$ et $l$ sont donnés, $w_{\mathrm{t}}$ est fixé. $L / l$ est proportionnel à $\sqrt{T_{0}}\left(\mathrm{cf}\right.$. (18)) et $w_{\mathrm{R}}$ à $1 / \tau^{2}$ (cf. (11)). Les droites $\eta_{c} w_{C}^{3}=$ cte dans la zone I et $w_{\mathrm{F}}=$ Cte dans la zone II sont des droites $\tau=$ cte. Nous pouvons donc effectuer une graduation directe du diagramme en $T_{0}$ et $\tau$. Nous avons tracé un tel diagramme sur la figure 6 en admettant que : le conducteur est en cuivre, $E=3,2 \times 10^{7} \mathrm{~V} / \mathrm{m}$ et $l=1 \mathrm{~mm}$ ou $1 \mathrm{~cm}$. Nous avons aussi admis $\gamma / \beta^{2}=1$. Rappelons que $\gamma$ est le rapport entre l'énergie de rupture $w_{\mathrm{R}}$ et l'énergie cinétique du morceau cassé et $\beta$ le rapport entre le temps $T$ de réisolement de l'interrupteur et le temps $\tau$ de transfert d'énergie ; aussi la valeur $\gamma / \beta^{2}=1$ est très plausible. Sur ce diagramme, nous pouvons donc lire directement les caractéristiques du fonctionnement correspondant à des points $C$ ou $F$.

Pour un point $D$, situé dans la zone $I^{\prime}$, la valeur de $\tau$ se lit dans la zone I. La valeur de $T_{0}$ n'est pas fixée de façon unique ; elle est comprise entre les deux valeurs lues respectivement dans les zones I et II.

Enfin, comme la durée $T_{0}$ de l'impulsion initiale de courant est toujours supérieure à $\tau$, nous avons tracé sur la figure 6 la courbe $T_{0} / \tau=1$ qui marque la limite extrême des possibilités de fonctionnement.

Notons qu'à la transition entre les zones I et II, définie par la demi-droite inférieure $\Omega \mathcal{C}$, les courbes ne sont plus du type $A C$ ou $A F H$ pur mais qu'elles sont intermédiaires. Aussi, la transition entre ces deux 


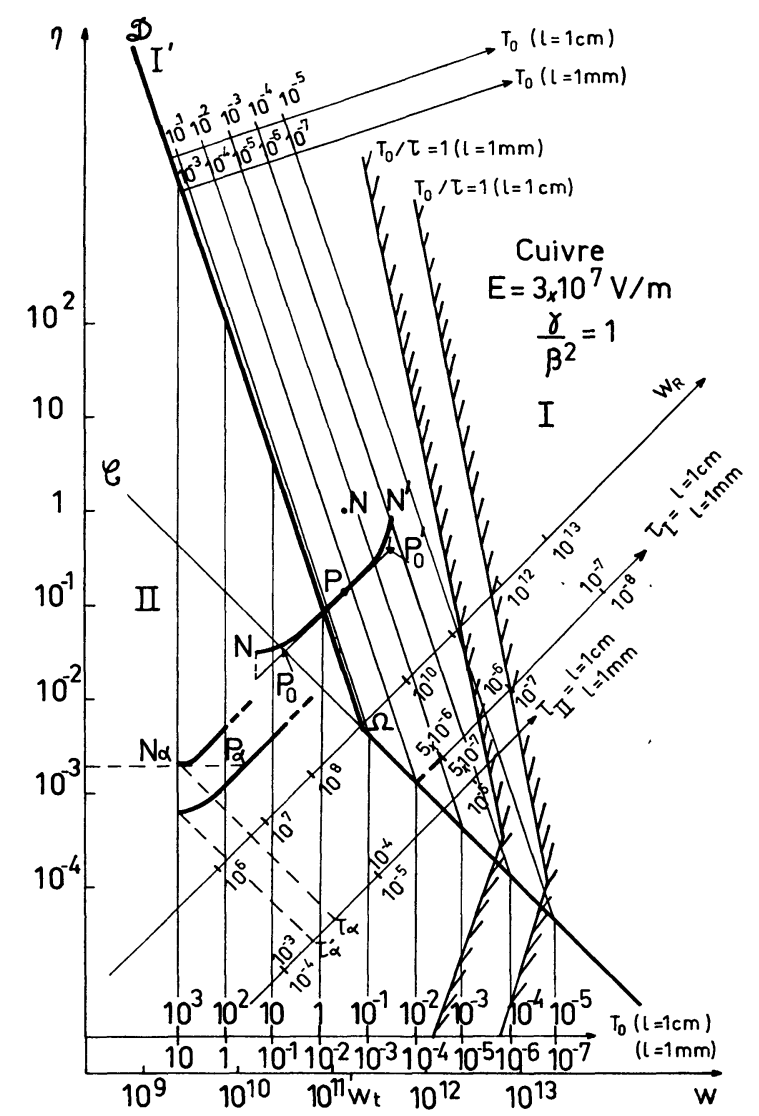

Fig. 6. - Diagramme en $T_{0}$ et $\tau$ pour les deux valeurs $1 \mathrm{~mm}$ et $1 \mathrm{~cm}$ de $l$. Lorsque le point $N$ de coordonnées $\left(T_{0}, \tau\right)$ est dans les zones $I$ ou $I^{\prime}$ ( $I^{\prime}$ est confondue avec $\Omega D$ ), il représente le meilleur point de fonctionnement pour ces valeurs des temps de stockage et de transfert d'énergie. Lorsque $N$ est dans la zone II, le point de fonctionnement est à choisir sur $N N^{\prime}$.

zones n'est-elle pas définie de façon parfaitement stricte.

Nous allons maintenant, à partir de ce diagramme, pouvoir préciser les possibilités des interrupteurs mécaniques.

5. Possibilités des interrupteurs mécaniques. 5.1 INFLUENCE DES DIVERS PARAMÈTRES PHYSIQUES. Le problème qui se pose généralement à un utilisateur se présente de la manière suivante : une impulsion initiale de courant de durée $T_{0}$ étant donnée, on veut effectuer le transfert d'énergie en un temps $\tau$. Comment faut-il procéder et quelles seront les performances obtenues ? Il est bien évident que si l'on trouve des pertes trop élevées, le problème ne possède pas de bonne solution.

Pour tésoudre ce problème, considérons à nouveau la figure 6 et portons-y le point caractéristique limite $N$ de coordonnées $T_{0}$ et $\tau$. (Rappelons que cette figure est tracée pour un ensemble de valeurs particulières de $l, E, \beta$ et $\gamma$, le conducteur étant du cuivre.) D'après ce que nous avons dit en 4.2:

- Si $N$ se trouve dans les zones I ou $\mathrm{I}^{\prime}$, le point de fonctionnement $P$ doit être confondu avec $N$.
- Si $N$ se trouve dans la zone II, les points de fonctionnement possibles $P$ sont situés sur un quasi-segment de droite d'extrémités $N$ et $N^{\prime}$, ce dernier point étant construit à partir de $N$ comme $H$ à partir de $G$ (cf. (33)). La coordonnée $X_{N}$ donne $w_{\mathrm{R}}\left(X_{N}=2 w_{\mathrm{R}}\right)$ et nous remarquerons que les valeurs de $\eta$ et $w$ augmentent lorsque $P$ se déplace de $N$ vers $N^{\prime}$.

Si l'on adopte pour les paramètres physiques des valeurs différentes de celles de la figure 6 , la graduation des axes de cette dernière varie de manière assez complexe. Aussi, nous allons maintenant examiner l'influence séparée des principaux paramètres; la longueur $l$, qui joue un rôle essentiel dans la détermination des performances, sera étudiée de manière détaillée.

a) Influence de la longueur de découpe $l$. - Le point $N$ est une fonction des divers paramètres physiques de la forme $\mathcal{L}=\mathfrak{L}\left(T_{0}, \tau, l, \Omega\right)$. Si l'on fait varier seulement $l$, le point $N=N(l)$ se déplace sur une courbe $\mathfrak{L}=\mathfrak{L}\left(T_{0}, \tau, \Omega\right)$ représentée sur la figure 7 . A chaque courbe $\mathfrak{L}=\mathfrak{L}\left(T_{0}, \tau, \Omega\right)$ est lié un ensemble de points $P$, eux-mêmes déterminés à partir des points $N=N(l)$. Parmi cet ensemble de points $P$, il en existe qui correspondent au "fonctionnement optimal ». Nous verrons qu'à ces points sont, à leur tour, associées des valeurs de $l$ optimales. Comme la notion de « fonctionnement optimal » est définie surtout par des considérations de bon sens (Existence de bornes

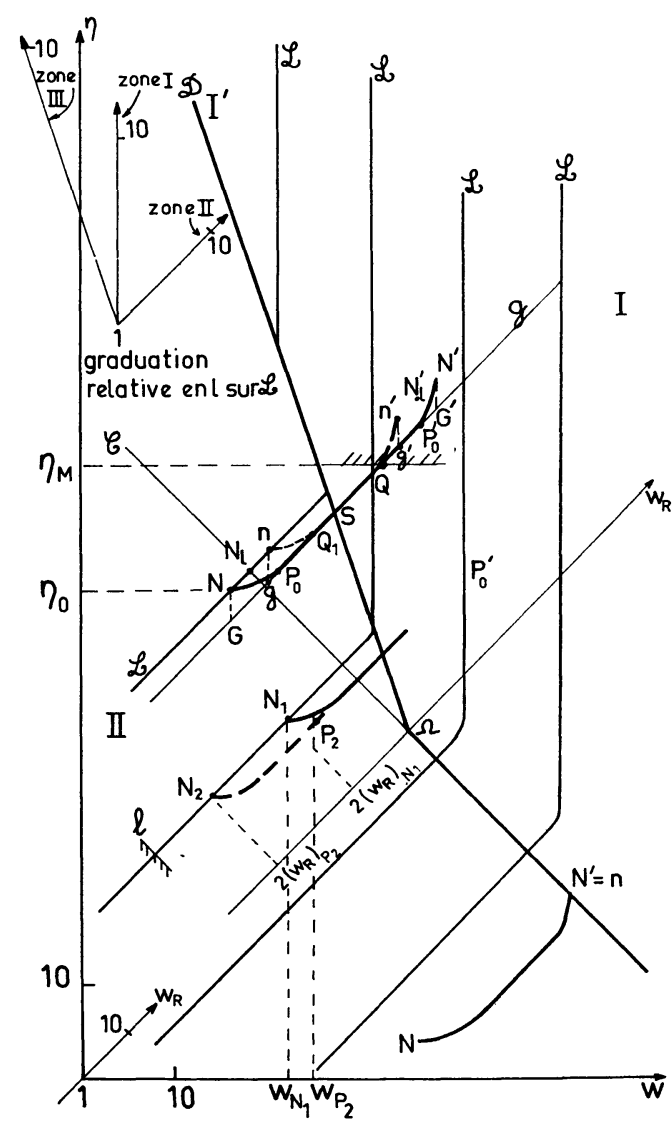

FIG. 7. - Courbes $\mathcal{L}$, lieux des points $N\left(T_{0}, \tau\right)$ lorsque $l$ varie, tracées pour diverses valeurs de $T_{0}$ et $\tau$. 
inférieure pour $l$ et supérieure pour $\eta-$ à pertes égales, choix de l'interrupteur le plus léger - etc.), il n'existe pas d'expression générale de la longueur optimale en fonction des paramètres du dispositif. Aussi, nous allons examiner les conditions pratiques de meilleur fonctionnement successivement pour chaque zone de la figure 7 .

Considérons donc un point $N$ situé dans la zone I. Nous avons vu plus haut que $P$ est alors confondu avec $N$. Laissant $T_{0}, \tau$ et $\Omega$ fixés, faisons varier $l$; $w$ reste constant tandis que $\eta$ varie comme $l^{2}$ (cf. (34)). Le " meilleur » point sera donc celui qui correspond à la plus petite dimension possible, c'est-à-dire celui qui est confondu avec le point le plus bas de la courbe $\mathcal{L}$ dans la zone I.

Si $N$ est dans la zone $\mathrm{I}^{\prime}$, on peut montrer que $\eta$ diminue avec $l$ alors que $w$ augmente ; il y a donc là encore intérêt à diminuer $l$ au maximum et à se placer à la limite inférieure de la zone $\mathrm{I}^{\prime}$.

Enfin, lorsque $N$ est situé dans la zone II, le choix du meilleur point de fonctionnement devient plus délicat. Tout d'abord, il faut remarquer que les valeurs de $w$ et $\eta=w_{\mathrm{R}} / w$ du point limite $N$ sont proportionnelles à $l((\mathrm{cf}$. (36)). Aussi, lorsque $l$ diminue, les pertes diminuent mais la densité d'énergie transférée diminue également ; le volume du conducteur rompu nécessaire pour transférer une énergie donnée croît donc (comme $1 / l)$. A priori, il faut donc choisir la dimension $l$ la plus grande possible compatible avec des pertes raisonnables. Cependant, il faut remarquer qu'il existe des points de fonctionnement $P$ possibles autres que les points limites $N$. Considérons à cet effet deux points $N_{1}$ et $N_{2}$ situés sur une même courbe $\mathcal{L}$ et correspondant à deux valeurs $l_{1}$ et $l_{2}$ de $l\left(l_{2}<l_{1}\right)$. Considérons aussi le point $P_{2}$ du quasi-segment $N_{2} N_{2}^{\prime}$ satisfaisant $\eta_{P_{2}}=\eta_{N_{1}}$. Nous avons alors en pratique

$$
w_{P_{2}} \# 2 w_{N_{1}} \text { et } \frac{\left(w_{\mathrm{R}}\right)_{N_{1}}}{\left(w_{\mathrm{R}}\right)_{P_{2}}} \#\left(\frac{l_{1}}{l_{2}}\right)^{2} \text {. }
$$

Le fonctionnement en $P_{2}$ est donc un peu meilleur qu'en $N_{1}$; l'énergie globale de rupture à mettre en œuvre en $P_{2}$ est aussi plus faible qu'en $N_{1}$ et cela d'autant plus que $l_{2}$ est petit. Aussi lorsque l'on cherche un point de fonctionnement correspondant à une valeur imposée $\eta=\eta_{N_{1}}$ de $\eta$, nous avons intérêt à choisir le point de fonctionnement $\boldsymbol{P}_{2}$, ce point étant obtenu avec $l_{2} \lesssim 0,7 l_{1}$. En ce qui concerne le choix précis de $l_{2}$, il reste assez libre ; cependant, des limitations pratiques à la construction de l'interrupteur imposent que sa dimension caractéristique reste supérieure à une valeur minimum $l_{0}$. Par suite il faudra vérifier $l_{0} \leqslant l_{2} \leqslant 0,7 l_{1}$.

Finalement, supposons que nous désirions construire un interrupteur pour lequel $T_{0}$ et $\tau$ sont donnés, les matériaux et $\beta^{2} / \gamma$ sont connus, la dimension minimale acceptable est $l_{0}$ et les pertes relatives maximales $\eta_{\mathbf{M}}$. L'étude que nous venons d'effectuer nous permet de répondre maintenant à la question fondamentale : est-ce possible ? Si la réponse est affirmative, quelle sera la dimension $l$ choisie et quelles seront les performances obtenues?

Pour répondre de manière précise à ces questions, nous allons procéder de la manière qui suit. Tout d'abord pour la valeur $l_{0}$, on trace le diagramme $\eta(w)$ et on y porte les graduations en $T_{0}$ et $\tau$ (la figure 6 représente ce diagramme lorsque $l_{0}=1 \mathrm{~mm}$ et $l_{0}=1 \mathrm{~cm}$ ). Dans ce diagramme, le couple des valeurs $\left(T_{0}, \tau\right)$ définit un point $N$ dont l'ordonnée est $\eta_{0}$. Comme le point $N$ est le point de fonctionnement limite, $\eta_{0}$ est la valeur minimale de $\eta$ associée au couple $\left(T_{0}, \tau\right)$ lorsque $l_{0}$ est donné. Si $\eta_{0}>\eta_{\mathrm{M}}$, il n'existe pas d'interrupteur répondant au problème posé. Supposons donc $\eta_{0}<\eta_{\mathrm{M}}$

Si $N$ est dans les zones I ou $\mathbf{I}^{\prime}$, nous savons que c'est le meilleur point de fonctionnement; on a $l=l_{0}$, $\eta=\eta_{0}$. On pourra éventuellement préférer prendre des dimensions $l>l_{0}$. Dans ce cas, on sait que le point $N$ se déplace sur une courbe $\mathcal{L}$ (cf. Fig. 7). Les nouvelles valeurs de $\eta$ et $w$ se comparent facilement à celles que l'on aurait obtenues pour $l=l_{0}$ à l'aide des graduations relatives de $l$ que l'on peut lire sur la figure 7 . Il faut cependant remarquer que $\eta$ croît très vite en $\left(l / l_{0}\right)^{2}$ dans la zone I et en $\left(l / l_{0}\right)^{3}$ dans la zone $I^{\prime}$.

Si le point $N$ est dans la zone II, le point de fonctionnement sera situé sur $N N^{\prime}$ (Fig. 7). Remarquons tout de suite sur la figure 7 que le fonctionnement en $P_{0}$ est préférable à celui en $N$ car $\eta_{P_{0}}$ est à peine supérieur à $\eta_{N}=\eta_{0}$ alors que $w_{P_{0}}$ est nettement plus grand que $w_{N}$. De même le fonctionnement en $P_{0}^{\prime}$ est préférable à celui en $N^{\prime}$. Dans le cas où $\eta_{\mathrm{M}} \sim \eta_{0}$, nous choisirons donc $P_{0}$ comme point de fonctionnement. Dans le cas où $\eta_{\mathrm{M}}>\eta_{P_{0}}$, appelons $Q$ le point de $P_{0} P_{0}^{\prime}$ tel que $\eta_{Q}=\eta_{\mathrm{M}}$ (nous prenons $Q$ confondu avec $P_{0}^{\prime}$ lorsque $\left.\eta_{\mathrm{M}}>\eta_{P_{0}^{\prime}}\right)$. Ce point $Q$ est alors le point de fonctionnement répondant le mieux au problème posé. Pour ce point $Q, l$ est indéterminé et il existe une infinité de quasi-segments $N N_{l}^{\prime}$ contenant $Q$. Parmi tous ces quasi-segments, il peut se trouver un segment limite $n n^{\prime}$ correspondant à la valeur $l_{n}$ de $l$ pour lequel $Q$ est soit du type $P_{0}$, soit du type $P_{0}^{\prime}$. Les valeurs permises de $l$ seront alors comprises entre $l_{0}$ et $l_{n}$ et le choix final sera lié à des considérations extérieures.

Pour déterminer $l_{n}$, indiquons la construction de $n$. Supposons tout d'abord $Y_{N}>2 Y_{\Omega}$. Alors, le quasisegment $N N^{\prime}$ (ou plutôt $N P_{0} P_{0}^{\prime} N^{\prime}$ ) coupe la droite $\mathfrak{D}$ en un point $S$ et l'on a $\mathbf{S G}=-\mathbf{S G}^{\prime}$ où $G$ et $G^{\prime}$ sont les projections de $N$ et $N^{\prime}$ sur la droite $\mathcal{G}$ confondue avec $P_{0} P_{0}^{\prime}$ (cf. (33)). Si $\eta_{\mathrm{M}}>\eta_{s}$, admettons que $Q$ est un point de type $P_{0}^{\prime}$; il est alors situé sur une courbe $n n^{\prime}, n^{\prime}$ s'obtenant à partir de $Q$ en traçant la courbe $Q n^{\prime}$ parallèle à $P_{0}^{\prime} N^{\prime}$ et $n$ à.partir de sa projection $g$ sur $\mathbf{G}$ par $\mathbf{S g}=-\mathbf{S g}^{\prime}$. ( $g^{\prime}$ est la projection de $n^{\prime}$ sur G.) La construction a été effectuée dans cette hypothèse sur la figure 7. Si $\eta_{M}<\eta_{S}$, nous admettons au contraire que $Q$ est un point de type $P_{0}$; il est alors situé sur une courbe $n n^{\prime}$ telle que $Q n$ soit parallèle à $P_{0} N$ (sur la figure 7 , nous avons appelé $Q_{1}$ le point 
conduisant à cette construction graphique). Ayant ainsi le point $n$, on lit facilement sur la figure le rapport $l_{n} / l_{0}$. Si, au contraire $Y_{N}<2 Y_{\Omega}$, la construction de $n$ reste la même si $T_{0}<\left(T_{0}\right)_{\Omega}$; sinon l'extrémité $N^{\prime}$ du quasi-segment $N N^{\prime}$ se trouve sur la droite $\mathcal{C}$ et $n$ est confondu avec $N^{\prime}$ (cf. Fig. 7).

$\beta)$ Influence de $\gamma / \beta^{2}$. - Dans les zones I et II, $l$ est toujours associé à $\gamma / \beta^{2}$ par l'intermédiaire du paramètre $\lambda=l \sqrt{\gamma / \beta^{2}}$. Un accroissement de $\sqrt{\gamma / \beta^{2}}$ équivaut donc à un accroissement de $l$ : l'étude apporte des conclusions identiques à celles que nous avons obtenues pour $l$.

Dans la zone $\mathrm{I}^{\prime}, \eta$ est proportionnel à $\gamma / \beta^{2}$ et $w$ est indépendant de cette quantité : on a donc en principe intérêt à choisir $\gamma / \beta^{2}$ le plus petit possible.

La valeur minimale de $\lambda$ ou de $\gamma / \beta^{2}$ correspond en particulier à la valeur minimale $\gamma_{0}$ de $\gamma$ (la quantité d'énergie de rupture $w_{\mathbf{R}}$ transformée en énergie cinétique du conducteur est alors maximale). Il est évident qu'il est toujours préférable de réaliser un coefficient $\gamma$ aussi petit que possible ; cependant, la discussion précédente montre que ce n'est pas toujours essentiel. De même, il est préférable mais pas toujours essentiel de vérifier $\beta=\beta_{0}$; (le temps de latence de la charge placée aux bornes de l'interrupteur mécanique est alors égal au temps de réisolement de cet interrupteur).

Notons que nous avons jusqu'ici considéré les pertes relatives réelles $\eta=\left(w_{\mathrm{R}}+w_{\mathrm{J}}\right) / w$. Cependant, on peut estimer dans certains cas que l'énergie de rupture n'a pas la même valeur pratique que l'énergie Joule dissipée. On introduit alors un coefficient $\mu_{\mathrm{r}}$ de pondération des pertes de rupture : les pertes relatives pondérées prennent alors la forme

$$
\eta_{P}=\mu_{\mathrm{R}} \frac{w_{\mathrm{R}}}{w}+\frac{w_{\mathrm{J}}}{w}
$$

Si l'énergie de rupture est peu coûteuse, $\mu_{R}<1$. Si elle est précieuse, $\mu_{\mathrm{R}}>1$. Dans tous les cas, cela équivaut à remplacer dans notre étude $\gamma$ par $\mu_{\mathrm{R}} \gamma$.

$\gamma)$ Influence des isolants et conducteurs employés. On montre aisément que l'on a toujours intérêt à employer l'isolant ayant la rigidité électrique la plus élevée possible. En ce qui concerne le métal rompu, il semble que les meilleurs matériaux sont le cuivre et l'aluminium. On montre que pour transférer la même énergie $w$ avec les mêmes pertes relatives $\eta$, la masse d'aluminium nécessaire est environ la moitié de celle du cuivre.

\subsection{PosSIBILITÉS LIMITES DES INTERRUPTEURS MÉCA-} NIQUES. - Cherchons maintenant quels sont les temps de transfert d'énergie $\tau$ les plus courts que nous puissions obtenir à partir d'une impulsion initiale de durée $T_{0}$. Nous fixons au départ les valeurs de $\beta^{2} / \gamma$, $\eta_{\mathrm{M}}$ et $l_{0} ; \eta$ et $l$ devront alors satisfaire les inéquations $\eta \leqslant \eta_{\mathrm{M}}$ et $l \geqslant l_{0}$.

Considérons tout d'abord le point limite $N_{\alpha}$ défini par $T_{0}, l$ et $\eta$; on peut lire sa coordonnée $\tau_{\alpha}$ sur la figure 6. Il est facile de voir que la valeur $\tau_{\alpha}^{\prime}$ correspondant à un point de fonctionnement quelconque $\boldsymbol{P}_{\alpha}$ ayant même $T_{0}$, même $l$ et même $\eta$ que $N_{\alpha}$ est toujours telle que $\tau_{\alpha}^{\prime}>\tau_{\alpha}$. Il en résulte que lorsque l'on recherche des temps courts, seuls les points limites $N$ sont à considérer. Par ailleurs, on peut montrer que les valeurs minimales de $\tau$ sont obtenues pour $\eta=\eta_{M}$ et $l=l_{0}$.

Nous avons porté sur la figure 8 les courbes $T_{0}(\tau)$ pour $l_{0}=1 \mathrm{~mm}$ et diverses valeurs de $\eta_{\mathrm{M}}$. Nous avons aussi indiqué sur cette figure le réseau des courbes $w=$ cte ; l'expression (6) nous a permis de graduer en $\beta^{2} w_{\mathrm{R}} / \gamma$ l'axe $O \tau$. Enfin, comme il n'est pas intéressant d'utiliser un interrupteur si $\tau$ n'est pas nettement plus petit que $T_{0}$, nous n'avons conservé que la partie de ce diagramme pour laquelle $T_{0}>10 \tau$.

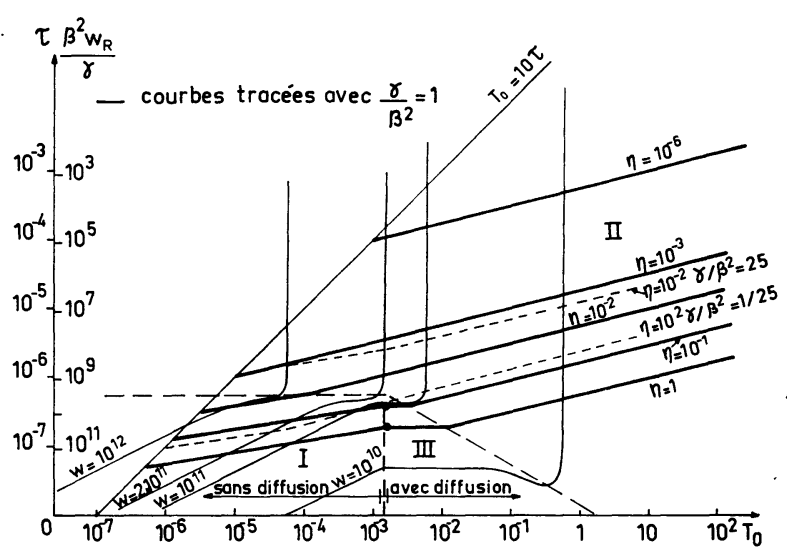

FIG. 8. - Valeur minimale de $\tau$ en fonction de $T_{0}$ lorsque le disjoncteur est en cuivre et que l'isolant est tel que

$$
E=3 \times 10^{7} \mathrm{~V} / \mathrm{m} \text {. }
$$

L'examen du diagramme permet de tirer les conclusions suivantes :

- Pour des rendements convenables $\left(\eta_{M} \lesssim 10^{-1}\right.$ ou $10^{-2}$ ) et des temps de passage initial du courant pas trop long $\left(T_{0} \lesssim 10^{-3}\right.$ ou $\left.10^{-4} \mathrm{~s}\right)$, il est possible d'atteindre pratiquement des temps de transfert d'énergie $\tau$ égaux ou sensiblement inférieurs à $1 \mu \mathrm{s}$.

- Les temps $\tau$ minimaux dépendent assez peu de $T_{0}$ $\left(\tau \sim T_{0}^{-1 / 6}\right.$ dans la zone $\mathrm{I}$, et $\tau \sim T_{0}^{-1 / 4}$ dans la zone II).

- Les temps les plus courts s'obtiennent avec une valeur relativement fixée et assez modérée de l'énergie de rupture $\left(w_{\mathbf{R}} \simeq 10^{10} \mathrm{~J} / \mathrm{m}^{3}\right.$ pour des valeurs $\eta_{\mathbf{M}}$ convenables).

6. Conclusion. - L'étude que nous venons d'effectuer était destinée à déterminer les performances des interrupteurs dits à rupture mécanique, dans lesquels on remplace très rapidement un morceau de métal de dimension caractéristique $l$ par un isolant injecté brutalement entre les lèvres de la coupure.

Nous avons tout d'abord montré qu'un transfert d'énergie ne pouvait être effectué rapidement que si 
l'impédance de la charge augmentait rapidement après le début de l'ouverture du disjoncteur.

Par ailleurs, si l'on se donne le temps $T_{0}$ de passage initial du courant, le temps $\tau$ de transfert d'énergie, la dimension caractéristique minimale $l_{0}$ ainsi que les pertes relatives maximales $\eta_{\mathbf{M}}$ que l'on accepte, on peut alors déterminer à partir des figures 6 et 7 par la construction définie à la fin du paragraphe $5.1 \alpha$, l'existence ou non d'un interrupteur acceptant ces données. Si cet interrupteur existe, la construction permettra de déterminer ses performances en ce qui concerne les pertes relatives, l'énergie transférable par unité de volume de conducteur, l'énergie de rupture et la dimension à choisir.
Notre étude nous a enfin permis de dégager l'ensemble des conclusions globales suivantes :

- Les meilleurs conducteurs à rompre paraissent être le cuivre et l'aiuminium qui conduisent à des performances sensiblement équivalentes.

- Les performances croissent proportionnellement aux qualités d'isolement de l'isolant injecté.

- La dimension caractéristique $l$ doit être prise en général aussi petite que possible.

- Si de l'énergie est stockée pendant un temps pouvant atteindre la ms, elle peut être transférée en un temps inférieur à la $\mu$ s avec un rendement convenable.

\section{Bibliographie}

[1] Heroin, P., Benoist, C., Rev. Gén. d'Electricité 80 (1971) 863.

[2] SolÉ, J., Entropie no 39, 21, 1971.

[3] Maldy, J., Ferrier, M., Santmaria, E., Electrotechnica 19 (1972) 637.

[4] Reece, N. P., Proc. I. E. E. E., 110 (1963) 803.

[5] Holmes, R., Yanabu, S., J. Phys. D 6 (1973) 1217.

[6] Vedel, J. et al., Rev. Gén. d'Electricité 80 (1971) 873.

[7] Di Marco, J. N., Burkhardt, L. C., J. Appl. Phys. 41 (1973) 3894.

[8] Antoni, B., Revue Phys. Appl. 9 (1974) 331.
[9] Damidau, F., Rioux, C., C. R. Hebd. Séan. Acad. Sci. 270 (1970) 1384.

[10] Damidau, F., Delmas, A., Hochard, L., Rioux, C., Proceedings of the VII Symposium on fusion technology. Grenoble 24/27 octobre 1972, édité par " Centre for Information and Documentation - Luxembourg December 1972".

[11] Rioux, C., Rioux-Damidau, F., Rapport interne LF $\mathrm{n}^{\circ} 12$, juin 1973.

[12] LANDAU et Lifschitz, Mécanique des fluides. Editions (Mir, Moscou) Chap. V.

[13] Somon, J. P., Thèse de Doctorat ès sciences (Orsay 1968). 